\title{
Clinical Impact, Safety, and Efficacy of Single- versus Dual-Coil ICD Leads in MADIT-CRT
}

\author{
VALENTINA KUTYIFA, M.D., PH.D., M.Sc., ${ }^{*}, \dagger, \#$ ANNE-CHRISTINE HUTH \\ RUWALD, M.D. $,{ }^{*}, \ddagger$,\# MEHMET K. AKTAS, M.D., ${ }^{*}$ CHRISTIAN JONS, M.D., Ph.D. $\ddagger$ \\ SCOTT McNITT, M.S., ${ }^{*}$ BRONISLAVA POLONSKY, M.S., ${ }^{*}$ LASZLO GELLER, M.D., PH.D., $\dagger$ \\ BELA MERKELY, M.D., Ph.D., † ARTHUR J. MOSS, M.D., ${ }^{*}$ WOJCIECH ZAREBA, M.D., PH.D., ${ }^{*}$ \\ and POUL ERIK BLOCH THOMSEN, M.D., PH.D.§
}

From the ${ }^{*}$ University of Rochester Medical Center, Heart Research Follow-Up Program, Rochester, NY, USA; †Semmelweis University Heart Center, Budapest, Hungary; †Gentofte University Hospital, Department of Cardiology, Copenhagen, Denmark; and $§$ Aalborg University Hospital, Department of Cardiology, Aalborg, Denmark

\begin{abstract}
Single- versus Dual-Coil ICD Leads. Background: Current data on efficacy, safety and impact on clinical outcome of single- versus dual-coil implantable cardioverter-defibrillator (ICD) leads are limited and contradictory.

Methods: Defibrillation threshold (DFT) at implantation and first shock efficacy were compared in patients implanted with single- versus dual-coil ICD leads in MADIT-CRT. The risk for atrial tachyarrhythmias and all-cause mortality were evaluated. Short- ( $<30$ days after the implantation) and long-term (throughout the entire study duration) complications were assessed.

Results: Patients with dual-coil ICD leads had significantly lower DFTs compared to patients with singlecoil ICD leads $(17.6 \pm 5.8 \mathrm{~J}$ vs $19.4 \pm 6.1 \mathrm{~J}, \mathrm{P}<0.001)$. First shock efficacy was similar among patients with dual and single-coil ICD leads $(89.6 \%$ vs $92.3 \%, P=1.00)$. When comparing patients with dual versus single-coil ICD leads, there was no difference in the risk of atrial tachyarrhythmias (HR $=1.57,95 \%$ CI: 0.81-3.02, $\mathrm{P}=\mathbf{0 . 1 8})$, or in the risk of all-cause mortality $(\mathrm{HR}=1.10,95 \% \mathrm{CI}: 0.58-2.07, \mathrm{P}=0.77)$. Patients implanted with single- or dual-coil ICD lead had similar short and long-term complication rates (short-term HR $=0.96,95 \%$ CI: $0.56-1.65, P=0.88$, long-term procedure-related HR $=0.99,95 \%$ CI: $0.62-1.59, P=1.00$, long-term $\mathrm{ICD}$ lead related: $\mathrm{HR}=1.2,95 \% \mathrm{CI}$ : $0.5-2.9, \mathrm{P}=0.68$ ) during the mean follow-up of 3.3 years.

Conclusions: Patients with single-coil ICD leads have slightly higher DFTs compared to those with dualcoil leads, but the efficacy, safety, and clinical impact on atrial tachyarrhythmias, and mortality is similar. Implantation of single-coil ICD leads may be favorable in most patients. (J Cardiovasc Electrophysiol, Vol. 24, pp. 1246-1252, November 2013)
\end{abstract}

atrial arrhythmias, heart failure, implantable cardioverter defibrillators, MADIT-CRT mortality

\section{Background}

With the technological development of cardiac device therapy, the implantation of dual-coil implantable cardioverter defibrillator (ICD) leads has become widespread, possibly due to the reported lower defibrillation threshold compared to single-coil ICD leads. ${ }^{1-8}$ However, small differences in defibrillation threshold are less likely to be clinically meaningful with today's high energy ICD devices. ${ }^{9}$ Some

\#Both authors contributed equally to the work.

The MADIT-CRT study was supported by a research grant from Boston Scientific, St. Paul, Minnesota, to the University of Rochester School of Medicine and Dentistry.

Address for correspondence: Valentina Kutyifa, M.D., Ph.D., M.Sc., Heart Research Follow-Up Program, University of Rochester Medical Center, 265 Crittenden Blvd. CU 420653, Rochester, NY 14642, USA. Fax: +1-585273-5283; E-mail: valentina.kutyifa@ heart.rochester.edu

Manuscript received 19 January 2013; Revised manuscript received 26 May 2013; Accepted for publication 28 May 2013.

doi: $10.1111 /$ jce. 12219 studies also suggested that defibrillation threshold does not reflect therapy efficacy (shock conversion rate) during the follow-up. ${ }^{10}$ Furthermore, dual-coil ICD leads have more complicated lead structure that might be associated with higher risk of lead-related complications including lead dislodgement, insulation failure or lead fracture that need lead extraction. ${ }^{11}$

We hypothesized that the implantation of dual-coil ICD leads may result in more atrial tachyarrhythmias when compared to single-coil ICD leads, possibly due to mechanical irritation of the right atrium caused by the superior vena cava (SVC) coil. We also hypothesized that dual-coil ICD lead implantations bear higher complication rates when compared to single-coil ICD leads. To our knowledge, there have been no large cohort clinical studies investigating the clinical outcome of atrial tachyarrhythmias and all-cause mortality in patients with single- and dual-coil ICD leads. Therefore, the aim of our study was to evaluate the clinical impact, efficacy, and safety of single- versus dual-coil ICD leads by evaluating the defibrillation threshold, complication rate, and first shock efficacy, and to assess the risk of atrial tachyarrhythmias and all-cause mortality in patients receiving single- versus dualcoil ICD lead systems, enrolled in MADIT-CRT. 


\section{Methods}

\section{MADIT-CRT}

The protocol and primary results of the MADIT-CRT study have previously been published. ${ }^{12,13}$ Briefly, 1,820 patients with ischemic or nonischemic cardiomyopathy, an ejection fraction $\leq 30 \%$ and a prolonged interventricular conduction delay with QRS complexes $>130$ milliseconds were randomized in a 3:2 fashion to CRT-D or ICD. Patients were excluded from the study if they had an existing indication for implantation with CRT-D, NYHA class III or IV or myocardial infarction within 90 days prior to enrollment, or those with permanent atrial fibrillation, or paroxysmal atrial fibrillation at the time of enrollment.

The study was conducted in the period from December 22, 2004 through June 24, 2009, at which time it was stopped by the recommendation of the data safety monitoring board. An extended follow-up was conducted until September 10, 2010.

This study comprised all patients who received an ICD or CRT-D device in MADIT-CRT $(\mathrm{n}=1,790)$. We excluded 7 patients who had a subcutaneous array implanted during the implantation procedure $(\mathrm{n}=1,783)$. Three patients had a single-coil RV lead implanted together with a SVC coil; they were categorized as dual-coil patients.

\section{Device Programming and Interrogation}

Commercially available ICD and CRT-D devices from Boston Scientific were used in this trial and standard transvenous mechanisms for implantation were followed. The implantation of a single- or dual-coil ICD lead was left to the discretion of the implanting physician. ICD and CRT-D devices were programmed according to the prespecified protocol, ${ }^{12}$ having a ventricular tachycardia (VT) zone programmed at 180 beats per minute (bpm) and the ventricular fibrillation (VF) zone at $210 \mathrm{bpm}$. Sensitivity was set at the physician's discretion. The detection duration was recommended to be 2.5 seconds in the VT zone and 1.0 seconds in the VF zone according to current standards.

The protocol suggested programming the first VT zone therapy to burst-type antitachycardia pacing (ATP) with 8 pulses at $88 \%$ of the measured cycle length with a $10 \mathrm{~ms}$ decrement between bursts, and shock therapy at the defibrillation threshold plus at least $10 \mathrm{~J}$, then maximum energy biphasic shocks.

All ICD and CRT-D devices were interrogated 1 month after enrollment and every 3 months thereafter until the end of the trial. All interrogation discs were sent to an independent core-laboratory for final adjudication.

\section{Defibrillator Threshold Testing}

There was no DFT testing protocol described for patients enrolled in MADIT-CRT and the decision whether or not to perform DFT testing was left to the discretion of the implanting physician. Additionally, it was not specified if the enrolling centers should use the stepwise approach or an empiric value to evaluate the defibrillation threshold. As a result, a variety of different methods were observed to establish the baseline defibrillation threshold. However, the majority of patients $(91 \%)$ did undergo DFT testing within 1 month of device implant. Prior results have shown that the method of
VF induction during DFT testing does not affect the DFT test results. $^{14}$

\section{Endpoints}

The endpoints of the current analysis comprised defibrillator threshold (DFT) testing data at implantation, short-term ( $<30$ days) and long-term complication rates, and the occurrence of atrial tachyarrhythmias and all-cause mortality during the follow-up.

Short-term procedure-related complications were analyzed from the reported adverse events and defined as any complication occurring within the first 30 days after implantation that required either active resuscitation (cardiac arrest, VT/VF or respiratory arrest) or invasive procedures due to complications such as pneumothorax, hemothorax, coronary dissection, tamponade, atrial perforation, infection needing surgery, hematoma or hemorrhage.

Long-term right ventricular lead related complications were captured throughout the entire duration of the study and comprised lead dislodgement, insulation failure, lead fracture, high ventricular threshold, coil fracture, over- or undersensing and postimplant myocardial perforation.

First shock efficacy, defined as a first spontaneous clinical occurrence of a VT or VF episode where 1 shock successfully terminated the ventricular arrhythmia, was assessed from independently adjudicated appropriate therapy episodes from device interrogation electrograms and defined as 1 appropriate ICD shock in a VT or VF episode.

Atrial tachyarrhythmias were captured from the ICDs during follow-up and adjudicated by an independent adjudication committee from the EGMs and comprised atrial fibrillation, atrial flutter, regular supraventricular tachycardia, and atrial tachycardia events. Combined assessment of atrial fibrillation and atrial flutter was considered as a separate end point.

Ventricular arrhythmias were also captured from the ICDs during follow-up and adjudicated by an independent adjudication committee using the EGMs of the episodes.

All-cause mortality was defined as death from any cause, and was captured during the entire follow-up of the study and adjudicated by an independent mortality committee blinded to clinical data or treatment assignment.

\section{Statistical Analysis}

Patients were categorized into 2 groups based on whether a single- or dual-coil ICD lead was implanted. Baseline characteristics were compared between patient subgroups using nonparametric Wilcoxon or Kruskal-Wallis tests for continuous variables and chi-square test for dichotomous variables, as appropriate. Defibrillation threshold test results were compared between the patient subgroups using the KruskalWallis test. The first shock efficacy was compared using chi-square test and Fisher's exact test.

Cumulative probability of atrial tachyarrhythmia episodes, atrial fibrillation/flutter episodes, and all-cause mortality were displayed according to the Kaplan-Meier method, with comparisons of cumulative event rates by the log-rank test.

Multivariate Cox proportional hazard's regression analyses were performed to estimate the risk of dual- versus single-coil ICD lead on atrial tachyarrhythmias, atrial fibrillation/flutter, all-cause mortality and complications. In the 
TABLE 1

Baseline Clinical Characteristics of Patients with Single- versus Dual-Coil ICD Leads

\begin{tabular}{lccc}
\hline $\begin{array}{l}\text { Clinical } \\
\text { Characteristics }\end{array}$ & $\begin{array}{c}\text { Single-Coil RV } \\
\text { Leads (n= 162) }\end{array}$ & $\begin{array}{c}\text { Dual-Coil RV } \\
\text { Leads (n= 1,621) }\end{array}$ & P Value \\
\hline $\begin{array}{l}\text { Median follow-up } \\
\text { time (months) }\end{array}$ & 35.1 & 40.5 & $<\mathbf{0 . 0 0 1}$ \\
Female & $50(31)$ & $395(24)$ & 0.068 \\
Age at enrollment & $63.7 \pm 9.8$ & $64.6 \pm 10.7$ & 0.176 \\
CRT-D treatment & $97(60)$ & $978(60)$ & 0.910 \\
Ischemic NYHA & $17(10)$ & $243(15)$ & 0.122 \\
$\quad$ class I & & & \\
Ischemic NYHA & $55(34)$ & $665(41)$ & 0.080 \\
$\quad$ class II & & & \\
Nonischemic & $90(56)$ & $713(44)$ & $\mathbf{0 . 0 0 5}$ \\
$\quad$ NYHA class II & & & \\
QRS & $160.6 \pm 18.6$ & $157.8 \pm 19.8$ & $\mathbf{0 . 0 2 7}$ \\
LBBB & $129(80)$ & $1,130(70)$ & $\mathbf{0 . 0 0 8}$ \\
RBBB & $18(11)$ & $205(13)$ & 0.571 \\
IVCD & $15(9)$ & $284(18)$ & $\mathbf{0 . 0 0 7}$ \\
BMI & $28.6 \pm 5.2$ & $28.7 \pm 5.3$ & 0.954 \\
Diabetes & $42(26)$ & $500(31)$ & 0.191 \\
Hypertension & $89(55)$ & $1,045(65)$ & $\mathbf{0 . 0 1 4}$ \\
Prior MI & $51(31)$ & $709(45)$ & $\mathbf{0 . 0 0 1}$ \\
GFR & $71.4 \pm 20.5$ & $69.0 \pm 20.2$ & 0.217 \\
Antiarrhythmic & $9(6)$ & $138(9)$ & 0.192 \\
$\quad$ drug treatment & & & \\
LVEF & $29.5 \pm 3.5$ & $29.0 \pm 3.4$ & $\mathbf{0 . 0 3 4}$ \\
LVEDV indexed & $125.2 \pm 30.3$ & $123.0 \pm 27.6$ & 0.565 \\
$\quad$ by BSA & & & \\
LVESV indexed & $88.8 \pm 24.5$ & $87.8 \pm 22.3$ & 0.931 \\
by BSA & & & \\
LAV indexed by & $45.8 \pm 10.9$ & $46.7 \pm 9.9$ & 0.132 \\
$\quad$ BSA & & & \\
\hline Vat & & & \\
\hline
\end{tabular}

Values are given as the percentage of patients or mean \pm SD. NYHA $=$ New York Heart Association class; BMI = body mass index; IVCD = intraventricular conduction delay; GFR $=$ glomerular filtration rate; $\mathrm{LAV}=$ left atrial volume; $\mathrm{LBBB}=$ left bundle branch block; LVEDV = left ventricular end-diastolic volume; $\mathrm{LVEF}=$ left ventricular ejection fraction; LVESV = left ventricular end-systolic volume; $\mathrm{MI}=$ myocardial infarction; $\mathrm{RBBB}=$ right bundle branch block.

multivariate model we adjusted for relevant variables selected by best subset regression analysis. The analysis was stratified by the location of the enrolling center (outside/inside USA). A 2-tailed $\mathrm{P}$ value below or equal to 0.05 was considered statistically significant. All analyses were conducted using SAS statistical software 9.3 version (SAS Institute, Cary, NC, USA).

\section{Results}

\section{Baseline Clinical Characteristics}

Baseline clinical characteristics of patients with singlecoil ICD lead $(n=162)$ versus dual-coil ICD lead $(n=1,621)$ are depicted in Table 1. Patients implanted with a single-coil ICD lead had more often nonischemic cardiomyopathy, wide QRS duration, LBBB, hypertension, prior MI and less often an IVCD ECG pattern. Baseline left ventricular ejection fraction (LVEF) was slightly higher in the single-coil ICD lead patient group compared to patients implanted with a dual-coil ICD lead. The proportion of CRT-D implantation was similar in both subgroups $(60 \%)$. Medical treatment, including antiarrhythmic drugs, was similar across the subgroups.

We found a substantial difference in the usage of singleversus dual-coil ICD leads by the location of the enrolling
TABLE 2A

Defibrillation Threshold Testing Results in Patients with Implanted Singleand Dual-Coil ICD Leads

\begin{tabular}{lccc}
\hline & \multicolumn{2}{c}{ Defibrillation Threshold } & \\
\cline { 2 - 3 } & Single-Coil Leads & Dual-Coil Leads & P Value \\
\hline Mean \pm SD & $19.4 \pm 6.1$ & $17.6 \pm 5.8$ & $<\mathbf{0 . 0 0 1}$ \\
\hline & & & \\
\hline
\end{tabular}

TABLE 2B

First Shock Efficacy (1 Single Shock Terminates VT or VF) in Patients with Implanted Single- and Dual-Coil ICD Leads

\begin{tabular}{lccc}
\hline & \multicolumn{2}{c}{ First Shock Efficacy } & \\
\cline { 2 - 3 } & Single-Coil Leads & Dual-Coil Leads & P Value \\
\hline Events/patients & $12 / 13$ & $146 / 163$ & \\
Percentage & $92.3 \%$ & $89.6 \%$ & $1.000^{*}$ \\
\hline
\end{tabular}

*Fisher's exact test.

centers. More single-coil leads were implanted outside of the USA ( $66 \%$ vs $34 \%, \mathrm{P}<0.001)$. There have been 82 of 110 centers (75\%) using dual-coil ICD leads exclusively. Out of the 87 US centers, 17 centers implanted a total of 55 singlecoil ICD leads (1-12 leads, $2-22 \%$ of all ICD leads per site). Out of the 23 OUS centers, 11 sites implanted 107 single-coil ICD leads (1-29 leads, 1-27\% of all ICD leads per center).

\section{Defibrillator Threshold Testing, First Shock Efficacy}

Defibrillator threshold testing was performed in 1,503 patients (93\%) with dual-coil ICD leads and in 149 patients (92\%) with single-coil ICD leads, within 1 month of the device implantation.

Patients implanted with a dual-coil ICD lead had a significantly lower defibrillation threshold than those with a single-coil ICD lead (dual coil 17.6 $\pm 5.8 \mathrm{~J}$, vs single coil $19.4 \pm 6.1 \mathrm{~J}(\mathrm{P}<0.001)$ (Table $2 \mathrm{~A})$. However, the difference was only an average of $1.8 \mathrm{~J}$ between single- and dual-coil ICD lead patients.

Evaluating the efficacy of first shock, we found no difference in patients implanted with single- or dual-coil ICD leads (single coil 92.3\% vs dual coil 89.6\%, $\mathrm{P}=1.00$ ) (Table $2 \mathrm{~B}$ ).

\section{Short- and Long-Term Complications}

Procedure-related complications within 30 days of implantation occurred in 16 of $162(10 \%)$ patients with singlecoil and in 122 of $1,621(8 \%)$ patients with dual-coil ICD leads. Right ventricular (RV) lead related complications during the mean follow-up time of 40 months occurred in 6 patients with single-coil ICD leads (3.7\%) and in 51 patients with dual-coil ICD leads (3.2\%). A total of 71 patients had their RV lead explanted, 66 patients $(3.7 \%)$ in the dual-coil ICD lead group and 5 patients $(3.1 \%)$ in the single-coil ICD lead group. There was no significant difference in the crude event rates in both short-and long-term complications between the subgroups, as shown in Table 3.

In the multivariate Cox proportional regression model, adjusting for gender, ischemic heart disease and enrolling center there was no difference in short- and long-term procedurerelated complication or in long-term RV-lead related complications among patients implanted with single- or dualcoil ICD leads using single-coil patients as a reference 


\section{TABLE 3}

Short- and Long-Term Complications in Single- versus Dual-Coil ICD Lead Recipients

\begin{tabular}{lcc}
\hline & \multicolumn{2}{c}{ Complications } \\
\cline { 2 - 3 } & Single Coil & Dual-Coil \\
N = 162 & N= 1,621 \\
\hline Total Lead and Procedure-Related Complications within 30 Days \\
RV lead related & $2(1.2)$ & $19(1.2)$ \\
Procedure related & $16(9.9)$ & $122(7.5)$ \\
Individual Procedure-Related Complications & within 30 Days & \\
Cardiac arrest or VT/VF & $1(0.6)$ & $6(0.4)$ \\
Respiratory arrest & $1(0.6)$ & $4(0.3)$ \\
Coronary sinus dissection & 0 & $10(0.6)$ \\
Tamponade & $1(0.6)$ & $9(0.6)$ \\
Pneumothorax or hemothorax & $3(1.9)$ & $23(1.4)$ \\
Hematoma or hemorrhage & $10(6.2)$ & $60(3.7)$ \\
Arterial perforation & 0 & $2(0.1)$ \\
Infection needing operation & 0 & $17(1.1)$ \\
Long-Term Follow-Up & & \\
Any RV-lead related complications & $6(3.7)$ & $51(3.2)$ \\
RV lead explantations & $5(3.1)$ & $66(3.7)$ \\
Procedure-related complications & $21(13.0)$ & $171(10.6)$ \\
\hline
\end{tabular}

*Complications are listed as numbers and percentages, all $\mathrm{P}$ values are nonsignificant between the subgroups.

group (short-term procedure-related: $\mathrm{HR}=0.96,95 \% \mathrm{CI}$ : $0.56-1.65, \mathrm{P}=0.88$, interaction with treatment $\mathrm{P}=0.92$; long-term procedure-related: $\mathrm{HR}=0.99,95 \% \mathrm{CI}$ : $0.62-1.59$, $\mathrm{P}=1.00$, interaction with treatment $\mathrm{P}=0.79$; and long-term RV lead related: $\mathrm{HR}=1.2,95 \% \mathrm{CI}: 0.5-2.9, \mathrm{P}=0.68$, interaction with treatment $P=0.61$ ). Nonsignificant interaction with treatment $P$ values suggests that patients with single- or dual-coil ICD leads and an implanted CRT-D do not experience more short- or long-term complications compared to ICD patients with single- or dual-coil ICD leads.

There have been 71 (4\%) RV lead extractions during the mean study follow-up of 40 months (median: 41 months), but there was no significant difference in the proportion of lead extractions among those implanted with single- or dual-coil ICD leads.

\section{Atrial Tachyarrhythmias}

During the follow-up, 150 patients (9.3\%) developed atrial tachyarrhythmias implanted with dual-coil ICD leads, and 10 patients $(6.2 \%)$ with single-coil ICD leads $(P=0.247)$. Patients with single- versus dual-coil ICD leads had similar cumulative incidence of atrial tachyarrhythmias (3-year Kaplan-Meier cumulative event rate of 5\% in single-coil leads vs $9 \%$ in dual-coil leads, $\mathrm{P}=0.302$ ) (Fig. 1) as was the incidence of atrial fibrillation or flutter (3-year KaplanMeier cumulative event rate of $4 \%$ in both groups, $\mathrm{P}=0.94$ ) (data not shown).

Dual-coil ICD leads were not associated with a significantly increased risk of atrial tachyarrhythmias $(\mathrm{P}=0.180)$, or atrial fibrillation and atrial flutter $(\mathrm{P}=0.573)$ after adjustment for clinical covariates such as treatment, age at enrollment, gender, left bundle branch block (LBBB), ischemic etiology of cardiomyopathy, body mass index greater than 30 , coronary artery bypass graft surgery in the past, revascularization procedure in the past, baseline QRS duration, left ventricular ejection fraction at baseline, left atrial volume index at baseline. This was similar in patients with an

\section{TABLE 4}

Risk of Atrial Tachyarrhythmias, Atrial Fibrillation, and All-Cause Mortality in Single- versus Dual-Coil ICD Lead Patients

\begin{tabular}{|c|c|c|c|}
\hline Parameter & Hazard Ratio & $95 \% \mathrm{CI}$ & P Value \\
\hline \multicolumn{4}{|c|}{ All Atrial Tachyarrhythmias (156 Events/1,718 Patients) } \\
\hline Dual coil:single coil & 1.57 & $0.81-3.02$ & 0.180 \\
\hline \multicolumn{4}{|c|}{ Atrial Fibrillation And Atrial Flutter (85 Events/1,718 Patients) } \\
\hline Dual coil:single coil & 1.26 & $0.57-2.79$ & 0.573 \\
\hline \multicolumn{4}{|c|}{ All-Cause Mortality (176 Events/1,719 Patients) } \\
\hline Dual coil:single coil & 1.10 & $0.58-2.07$ & 0.771 \\
\hline \multicolumn{4}{|c|}{$\begin{array}{l}\text { * Model is adjusted for treatment, age at enrollment, gender, LBBB, ischemic } \\
\text { etiology of cardiomyopathy, body mass index greater than } 30 \text {, coronary } \\
\text { artery bypass graft surgery in the past, revascularization procedure in the } \\
\text { past, baseline QRS duration, left ventricular ejection fraction at baseline, } \\
\text { and left atrial volume index at baseline. The analysis was stratified by the } \\
\text { location of the enrolling center (outside/inside USA). }\end{array}$} \\
\hline
\end{tabular}

implanted ICD or CRT-D (interaction P value with treatment $>0.1$ for both endpoints) (Table 4).

\section{Effect of CRT-D on First Shock Efficacy and Ventricular Arrhythmias}

In patients with ventricular arrhythmias and single-coil ICD leads implanted, the first shock efficacy was $80 \%$ (8 of 10 patients) with an implanted ICD-only, and 100\% (5 of 5 patients) with an implanted CRT-D $(\mathrm{P}=0.524)$. In those implanted with dual-coil ICD leads, 67 of $79(85 \%)$ ICD patients and 78 of 82 (95\%) CRT-D patients had successful first appropriate shock during the follow-up $(\mathrm{P}=0.036)$.

In patients implanted with single-coil ICD lead, there was a significant reduction in the risk of ventricular tachyarrhythmias from CRT-D when compared to ICD-only patients $(\mathrm{HR}=0.38,95 \% \mathrm{CI}: 0.18-0.83, \mathrm{P}=0.015)$. The reduction in ventricular arrhythmias was also evident in patients implanted with dual-coil ICD leads and a CRT-D when compared to ICD-only patients (HR $=0.80,95 \%$ CI: $0.65-0.98$, $\mathrm{P}=0.028)$. When comparing patients implanted with a single-coil ICD lead to patients implanted with a dual-coil lead, we found a trend toward decreased risk of ventricular arrhythmias in patients implanted with a single-coil ICD lead $(\mathrm{P}=0.12)$.

\section{All-Cause Mortality}

During the study duration, 175 patients $(10.8 \%)$ died implanted with dual-coil ICD leads, and 12 patients (7.4\%) died implanted with single-coil ICD leads. The cumulative event rate was not significantly different among these patient subgroups $(\mathrm{P}=0.486)$ (Fig. 2). Consistent with this, implantation of a dual-coil ICD lead was not associated with an increased risk of mortality compared to a single-coil ICD lead after adjustment for relevant clinical covariates $(\mathrm{P}=$ 0.771) (Table 4). This was consistent in ICD and CRT-D patients (interaction $\mathrm{P}$ value with treatment $>0.1$ ).

\section{Sensitivity Analyses}

In patients with single-coil ICD leads there was a significantly shorter follow-up time, and this difference is coming from the fact that patients were more often implanted with single-coil ICD leads toward the end of the trial. The percentages of implanted single-coil ICD leads were $7.4 \%$ versus $6.2 \%$ versus $10.5 \%$ versus $17.8 \%$, respectively, for the years 


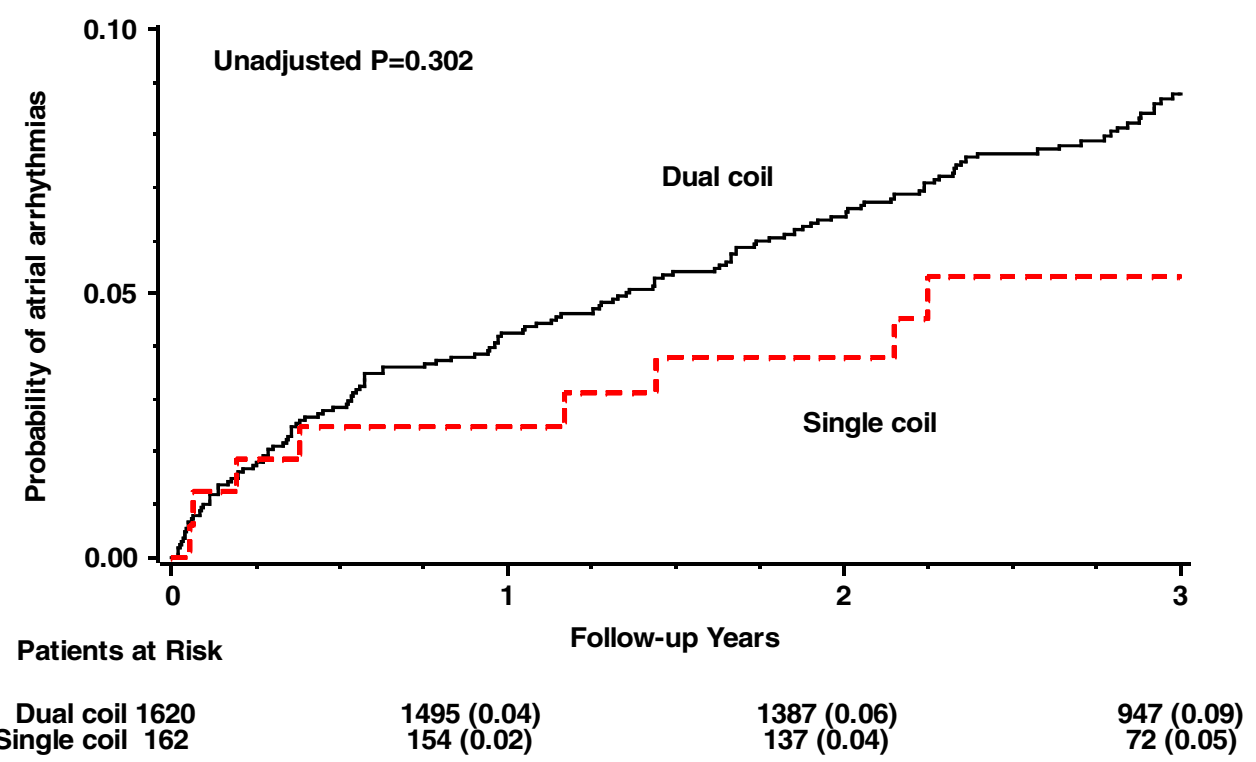

Figure 1. Atrial tachyarrhythmias in patients with single-versus dual-coil ICD leads.

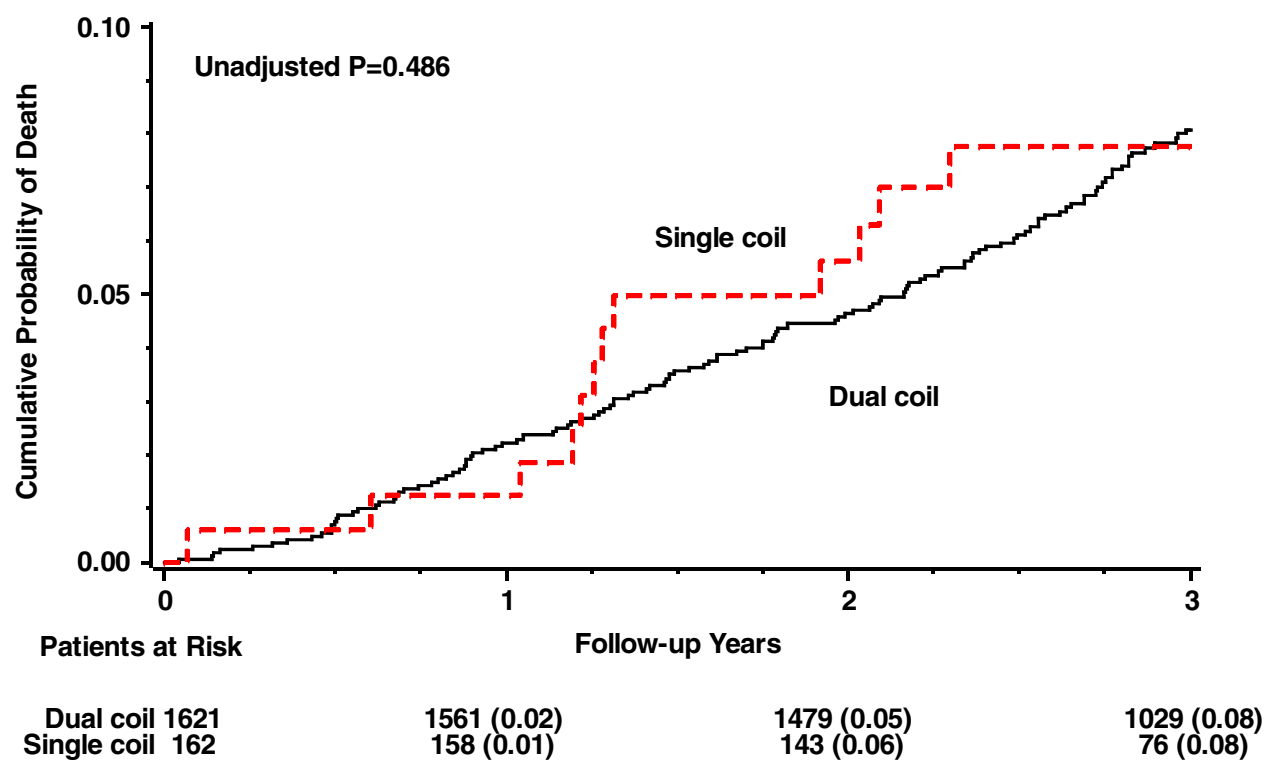

Figure 2. Cumulative probability of all-cause mortality in patients with single-, dual-coil ICD leads.

of 2005, 2006, 2007, and 2008. For sensitivity analyses we stratified the previous multivariate models for the year of enrollment and found consistent results: patients with dual-coil ICD leads had a trend toward a higher risk of atrial tachyarrhythmias $(\mathrm{HR}=1.59,95 \% \mathrm{CI}$ : $0.82-3.08, \mathrm{P}=0.166)$, but no difference was observed in atrial fibrillation or atrial flutter $(\mathrm{HR}=1.22,95 \% \mathrm{CI}: 0.55-2.74, \mathrm{P}=0.626)$ and in allcause mortality $(\mathrm{HR}=1.23,95 \% \mathrm{CI}: 0.65-2.34, \mathrm{P}=0.524)$.

\section{Discussion}

In this study, we demonstrated that ICD and CRT-D patients implanted with single- versus dual-coil ICD leads have similar complication rates during implantation and through the entire study follow-up. Furthermore, we did not find differences in atrial arrhythmia burden or all-cause mortality. However, we did see a trend toward a decreased risk of ven- tricular arrhythmias in patients implanted with a single-coil ICD lead compared to patients implanted with a dual-coil ICD lead. Patients implanted with dual-coil ICD leads had significantly lower energy requirement during DFT testing, but this difference was not associated with a better first shock efficacy during the follow-up.

To our knowledge, this is the first large cohort patient data comprehensively evaluating defibrillation threshold testing results, arrhythmia termination success, short- and long-term complications, mortality, and the incidence of atrial tachyarrhythmias in ICD and CRT-D patients implanted with single- versus dual-coil ICD leads.

Previous small trials did not show differences in DFT among patients with single- or dual-coil ICD leads. Schulte et al. ${ }^{6}$ randomized 83 patients to receive single- or dual-coil leads and found no difference in ICD energy requirements or the probability of successful defibrillation. Similar results 
were found by another group in patients with secondary or primary ICD indications (DFT single- vs dual coil, $10.2 \pm$ $5.2 \mathrm{~J}$ vs $10.3 \pm 4.1 \mathrm{~J}, \mathrm{P}=\mathrm{NS}){ }^{7}$

However, others reported significantly lower defibrillator thresholds in dual-coil ICD leads compared to single-coil ICD leads $(8.7 \pm 4.0 \mathrm{~J}$ vs $10.1 \pm 5.0 \mathrm{~J}, \mathrm{P}<0.02) .{ }^{4}$ These studies were conducted in small patient cohorts. In our analysis, we confirmed similar results in a large patient cohort. We found a statistically significant lower defibrillation threshold in dual-coil ICD leads versus single-coil ICD leads (mean value, single coil 19.4 J vs dual coil 17.6 J); however, the clinical value of a $1.8 \mathrm{~J}$ reduction in DFTs may be of limited significance especially with today's high energy ICDs that are capable of delivering up to $40 \mathrm{~J}$. This is further proven by our study results suggesting that the difference in the defibrillation threshold was not associated with a difference in the ICD shock efficacy among patients implanted with singleversus dual-coil ICD leads.

Furthermore, the actual need and benefit of defibrillator testing at implantation has recently been questioned. ${ }^{15-17}$ Michowitz et al. ${ }^{16}$ suggested that defibrillator testing in 256 CRT-D patients was associated with increased morbidity and no survival or device benefit during the follow-up. Another recent trial evaluated the effects of DFT testing in 2,120 consecutive patients and showed no significant differences in procedural complications or sudden cardiac death or resuscitation during the follow-up. ${ }^{18}$

Our study is unique in that we have evaluated the incidence of atrial tachyarrhythmias in single- versus dual-coil ICD recipients. We hypothesized that dual-coil ICD leads might be associated with more prevalent atrial arrhythmias because of the mechanical irritation of the left atrium caused by the SVC coil. Although our results did not support this hypothesis, there does appear to be a trend toward higher rates of both atrial and ventricular arrhythmias with dualcoil ICDs (adjusted P value $=0.18$ and 0.12 , respectively). However, the small sample size in the single-coil arm may have been underpowered to detect a statistically significant difference. Larger studies are warranted to further evaluate this association.

When evaluating the first shock efficacy in dual-coil ICDonly versus CRT-D patients, we found that in CRT-D patients the first shock in more frequently successful than in ICD patients. The reason for this association is unknown. The $\mathrm{P}$ value for the difference reached significance, however, because of the small sample size these data need to be interpreted with caution. Larger scale data are needed to further evaluate the possible advantage of dual-coil ICD leads in CRT-D patients compared to ICD patients.

Previous studies have not reported on complications in single- versus dual-coil ICD lead systems. It has also been questioned whether transvenous lead extraction of dual-coil leads could be associated with increased risk of mortality compared to single-coil lead systems due to a higher risk of complications associated with the extraction procedure. ${ }^{19}$ In our study, $71 \mathrm{RV}$ lead extractions were performed during a mean follow-up of 40 months, but there was no significant difference in the proportion of lead extractions among those implanted with single- or dual-coil ICD leads. Furthermore, we found no evidence for differences in all-cause mortality between single- and dual-coil ICD lead patients.

We were not able to show a significant difference between single- and dual-coil ICD leads with regard of the occurrence of short- and long-term procedure-related complications and long-term RV ICD lead-related complications.

In this analysis, we had a low number of single-coil ICD leads. Furthermore, we report a very low complication rate over a relatively short follow-up time. These factors might translate into limited statistical power in our analysis to detect significant differences in complication rate.

With the sample size available in our study, the power to detect a significant difference in outcomes such as complication rate or atrial arrhythmias, $7.5 \%$ versus $15 \%$ is $80 \%$, which would certainly represent a clinically meaningful difference for complication rates. The complication rates were generally much closer than this and so we feel confident that the lack of statistical significance reflects the true lack of difference in the complication rates between the 2 device types. For instance, the RV-lead related complication rates were $3.7 \%$ and $3.2 \%$ for single and dual coils, respectively. Ideally, a larger sample of patients with single-coil devices would give us more robust findings due to added statistical power. However, our data are to date the largest study sample from a randomized clinical trial suggesting that single-coil ICD leads are not inferior to dual-coil ICD leads. Further larger, randomized studies may be warranted to explore the efficacy, safety and the potential atrial proarrhythmic effect of dual-coil ICD leads.

Another possible limitation of our study is its nonrandomized fashion, which might contribute to potential bias. There was a difference of 5 months in the median follow-up time among patients implanted with single- versus dual-coil ICD leads. Furthermore, in MADIT-CRT, we do not have the programmed first shock energy available; therefore, the analysis on the first shock efficacy might be confounded. However, the defibrillation threshold was very low in both groups, and using the recommended $10 \mathrm{~J}$ safety margin, most patients might have had similar shock energies programmed.

\section{Conclusion}

In this analysis we suggest that patients implanted with single- versus dual-coil ICD lead have similar short- and long-term complication rates and there does not appear to be a difference in atrial arrhythmia burden and in all-cause mortality. Although patients with a dual-coil ICD system have a statistically significant lower energy requirement during DFT testing, this difference is not associated with better ICD treatment efficacy. Therefore, it may be reasonable to consider implantation of single-coil ICD systems in most patients, except when a higher defibrillation threshold is anticipated before implantation.

\section{References}

1. Bardy GH, Dolack GL, Kudenchuk PJ, Poole JE, Mehra R, Johnson G: Prospective, randomized comparison in humans of a unipolar defibrillation system with that using an additional superior vena cava electrode. Circulation 1994;89:1090-1093.

2. Kudenchuk PJ, Bardy GH, Dolack GL, Poole JE, Mehra R, Johnson G: Efficacy of a single-lead unipolar transvenous defibrillator compared with a system employing an additional coronary sinus electrode. A prospective, randomized study. Circulation 1994;89:2641-2644.

3. Gold MR, Foster AH, Shorofsky SR: Lead system optimization for transvenous defibrillation. Am J Cardiol 1997;80:1163-1167.

4. Gold MR, Olsovsky MR, Pelini MA, Peters RW, Shorofsky SR: Comparison of single- and dual-coil active pectoral defibrillation lead systems. J Am Coll Cardiol 1998;31:1391-1394. 
5. Gold MR, Olsovsky MR, DeGroot PJ, Cuello C, Shorofsky SR: Optimization of transvenous coil position for active can defibrillation thresholds. J Cardiovasc Electrophysiol 2000;11:25-29.

6. Schulte B, Sperzel J, Carlsson J, Schwarz T, Ehrlich W, Pitschner HF, Neuzner J: Dual-coil vs single-coil active pectoral implantable defibrillator lead systems: Defibrillation energy requirements and probability of defibrillation success at multiples of the defibrillation energy requirements. Europace 2001;3:177-180.

7. Rinaldi CA, Simon RD, Geelen P, Reek S, Baszko A, Kuehl M, Gill JS: A randomized prospective study of single coil versus dual coil defibrillation in patients with ventricular arrhythmias undergoing implantable cardioverter defibrillator therapy. Pacing Clin Electrophysiol 2003;26:1684-1690.

8. Gold M, Val-Mejias J, Leman RB, Tummala R, Goyal S, Kluger J, Kroll M, Oza A: Optimization of superior vena cava coil position and usage for transvenous defibrillation. Heart Rhythm 2008;5:394-399.

9. Ellis CR HJT: Single-coil versus dual-coil ICD lead shock efficacy in a large ICD registry. J Innovat Cardiac Rhythm Manag 2012;3:953-958.

10. Smits K, Virag N, Swerdlow CD: Impact of defibrillation testing on predicted ICD shock efficacy: Implications for clinical practice. Heart Rhythm 2013;10:709-717.

11. Neuzner J, Carlsson J: Dual- versus single-coil implantable defibrillator leads: Review of the literature. Clin Res Cardiol 2012;101:239-245.

12. Moss AJ, Brown MW, Cannom DS, Daubert JP, Estes M, Foster E, Greenberg HM, Hall WJ, Higgins SL, Klein H, Pfeffer M, Wilber D, Zareba W: Multicenter automatic defibrillator implantation trialcardiac resynchronization therapy (MADIT-CRT): Design and clinical protocol. Ann Noninvasive Electrocardiol 2005;10:34-43.
13. Moss AJ, Hall WJ, Cannom DS, Klein H, Brown MW, Daubert JP, Estes NA III, Foster E, Greenberg H, Higgins SL, Pfeffer MA, Solomon SD, Wilber D, Zareba W: Cardiac-resynchronization therapy for the prevention of heart-failure events. N Engl J Med 2009;361:1329-1338.

14. Zima E, Gergely M, Soos P, Geller LA, Nemes A, Acsady G, Merkely B: The effect of induction method on defibrillation threshold and ventricular fibrillation cycle length. J Cardiovasc Electrophysiol 2006;17:377381.

15. Stefano B, Pietro RR, Maurizio G, Maurizio L, Renato M, Pietro R, Alessandro P, Gianluca B, Monica M, Sergio C, Massimo S: Defibrillation testing during implantable cardioverter-defibrillator implantation in Italian current practice: The Assessment of Long-term Induction clinical ValuE (ALIVE) project. Am Heart J 2011;162:390-397.

16. Michowitz Y, Lellouche N, Contractor T, Bourke T, Wiener I, Buch E, Boyle N, Bersohn M, Shivkumar K: Defibrillation threshold testing fails to show clinical benefit during long-term follow-up of patients undergoing cardiac resynchronization therapy defibrillator implantation. Europace 2011;13:683-688.

17. Viskin S, Rosso R: The top 10 reasons to avoid defibrillation threshold testing during ICD implantation. Heart Rhythm 2008;5:391-393.

18. Brignole M, Occhetta E, Bongiorni MG, Proclemer A, Favale S, Iacopino S, Calo L, Vado A, Buja G, Mascioli G, Quartieri F, Tritto M, Parravicini U, Castro A, Tomasi C, Villani GQ, D’Acri MG, Klersy C, Gasparini M: Clinical evaluation of defibrillation testing in an unselected population of 2,120 consecutive patients undergoing first implantable cardioverter-defibrillator implant. J Am Coll Cardiol 2012;60:981-987.

19. Neuzner J, Carlsson J: Dual- versus single-coil implantable defibrillator leads: Review of the literature. Clin Res Cardiol 2012;101:239-245. 